\title{
LIPSCHITZ AND QUASICONFORMAL TUBULAR NEIGHBOURHOODS OF SPHERES IN CODIMENSION TWO
}

\author{
DAVID GAULD
}

In this paper it is shown that if $X$ is a codimension 2 sphere in $S^{n}, n \neq 4,5,6$, then $X$ has either a Lipschitz or a quasiconformal tubular neighbourhood if $X$ is either locally Lipschitz flat or locally quasiconformally flat.

The notation of this paper is the same as that established in [GV]. In particular $C$ denotes either of the categories LIP or QC. Theorem 3.3 of [GV] tells us that if, $X$ is a locally $C$-flat codimension 2 sphere in $S^{n}, n \neq 4,6$, and if $X$ is homotopically unknotted in $S^{n}$, then $\left(S^{n}, X\right)$ is $C$-homeomorphic to $\left(S^{n}, S^{n-2}\right)$. In this paper we consider the case where $X$ might be knotted, obtaining the following result.

Theorem 1. Let $X \subset S^{n}$ be a locally $C$-flat TOP $(n-2)$-sphere in $S^{n}$. If $n \neq 4,5$ or 6 then there is a neighbourhood $N$ of $S^{n-2}$ in $S^{n}$ and a C-embedding $\left(N, S^{n-2}\right) \rightarrow\left(S^{n}, X\right)$.

Analogously with Theorem 3.4 of [GV], we have the following result.

Theorem 2. Let $g: S^{n-2} \rightarrow S^{n}$ be a locally $C$-flat embedding. If $n \neq 4$ or 5 then $g$ extends to a $C$-embedding of a neighbourhood of $S^{n-2}$ in $S^{n}$.

Proof of Theorem 1. Encasing as in the proof of Theorem 3.3 of [GV], since $n \neq 6 \Rightarrow n-2 \neq 4$ we may assume that only two $C$-encasings are necessary to exhibit the local $C$-flatness of $X$.

Now transfer everything to $\overline{\boldsymbol{R}}^{n}$. Using the $C$-Schoenflies theorem we may extend one of the encasings to a $C$-homeomorphism of $\overline{\boldsymbol{R}}^{\boldsymbol{n}}$. If we replace $X$ by its inverse image under this homeomorphism, we see that it may be assumed that one of the two $C$-encasings is the inclusion. By reflection, we may assume that we have the following situation: $X \cap\left[\overline{\boldsymbol{R}}^{n} \backslash B^{n}(a)\right]=\overline{\boldsymbol{R}}^{n-2} \backslash B^{n-2}(a)$ for some $a<1$, and there is a $C$-embedding $h: B^{n} \rightarrow R^{n}$ with $h^{-1} X=B^{n-2}$ and $X \cap \bar{B}^{n} \subset h B^{n-2}$. Thus the knotted part of $X$ is trapped inside $B^{n}$ where it is encased by a single $C$-encasing. Assume that the norm on $\boldsymbol{R}^{n}$ is $\left|\left(x_{i}\right)\right|=\max \left\{\left|x_{i}\right|\right\}$ rather than the pythagorean norm so that $B^{n}$ is a cubic ball rather than a round ball, thus allowing PL methods.

Choose $\alpha:\left(V, \overline{\boldsymbol{R}}^{n-2}\right) \rightarrow\left(\overline{\boldsymbol{R}}^{n}, X\right)$, a topological embedding where $V$ is a neighbourhood of $\overline{\boldsymbol{R}}^{n-2}$ in $\overline{\boldsymbol{R}}^{n}$. The existence of $\alpha$ follows from the topological local flatness of $X$ : by $\left[\mathrm{KS}_{2}\right], X$ admits a normal disc bundle in $\overline{\boldsymbol{R}}^{n}$ since $n \neq 4$; as noted in [K], 
the 2-disc bundles are classified by $H^{2}(X ; Z)$ which, when $n \neq 4$, is the trivial group. Thus $X$ has a trivial normal disc bundle in $\overline{\boldsymbol{R}}^{n}$ thereby providing us with the embedding $\alpha$. Since $\alpha V$ is a neighbourhood of $\overline{\boldsymbol{R}}^{n-2} \backslash B^{n-2}=X \cap\left[\overline{\boldsymbol{R}}^{n} \backslash B^{n}\right]$, we may assume that $\overline{\boldsymbol{R}}^{n} \backslash B^{n} \subset \alpha V$ so, using the relative TOP-Schoenflies theorem, [B] and [GV], we may extend $\alpha \mid \overline{\boldsymbol{R}}^{n} \backslash B^{n}(b)$ for some $b \in(a, 1)$ to a homeomorphism $\beta$ of $\overline{\boldsymbol{R}}^{n}$ so that $\beta \overline{\boldsymbol{R}}^{n-2}=\overline{\boldsymbol{R}}^{n-2}$. Choose $r>0$ sufficiently small so that $\bar{B}^{n-2} \times B^{2}(r) \subset \beta^{-1} V$ and $\alpha \beta\left[\bar{B}^{n-2} \times B^{2}(r)\right] \subset h B^{n}$. Let $\gamma=\alpha \beta \mid \bar{B}^{n-2} \times B^{2}(r)$. Then the embedding $\gamma$ satisfies the following properties: im $\gamma \subset \bar{B}^{n} \cap h B^{n} ; \gamma$ is the identity on a neighbourhood of $S^{n-3} \times B^{2}(r) ; \gamma\left[B^{n-2} \times 0\right]=X \cap B^{n}$.

Suppose we can construct a $C$-embedding

$$
\delta: \bar{B}^{n-2} \times \bar{B}^{2}(r / 2) \rightarrow \bar{B}^{n}
$$

which is the identity on a neighbourhood of $S^{n-3} \times \bar{B}^{2}(r / 2)$ and satisfies $X \cap \bar{B}^{n} \subset$ $\delta\left[\bar{B}^{n-2} \times 0\right]$. Let

$$
N=\left[\overline{\boldsymbol{R}}^{n} \backslash \bar{B}^{n}\right] \cup\left[\bar{B}^{n-2} \times B^{2}(r / 2)\right],
$$

and extend $\delta$ over $N$ by the identity. Then $N$ is a neighbourhood of $\overline{\boldsymbol{R}}^{n-2}$ in $\overline{\boldsymbol{R}}^{n}$ and $\delta$ is a $C$-embedding. Moreover $\delta \overline{\boldsymbol{R}}^{n-2}=\left[\overline{\boldsymbol{R}}^{n-2} \backslash \bar{B}^{n-2}\right] \cup \delta\left[\bar{B}^{n-2} \times 0\right]=X$. Thus, apart from the change of scenery from $S^{n}$ to $\overline{\boldsymbol{R}}^{n}, \delta$ is the required $C$-embedding. Thus it is sufficient to construct the $C$-embedding $\delta$ as above.

Consider the TOP handle $\gamma$ : this is PL straight on $\partial \bar{B}^{n-2} \times B^{2}(r)=S^{n-3} \times B^{2}(r)$, being the inclusion there. Since $n \neq 4$ or 5 , either $n \leqq 3$ or $n-2 \neq 3$ and $n \geqq 5$. Using $[\mathrm{M}]$ in the former case and $\left[\mathrm{KS}_{1}\right]$ in the latter case, we may straighter $\gamma$. More precisely, there is an isotopy $\gamma_{t}: \bar{B}^{n-2} \times B^{2}(r) \rightarrow \bar{B}^{n}(0 \leqq t \leqq 1)$ with $\gamma_{0}=\gamma, \gamma_{1} \mid \bar{B}^{n-2} \times$ $\bar{B}^{2}(r / 2)$ PL and $\gamma_{t}=\gamma$ on a neighbourhood of

for some $s<r$. Let

$$
\left[S^{n-3} \times B^{2}(r)\right] \cup\left[\bar{B}^{n-2} \times\left(B^{2}(r) \backslash \bar{B}^{2}(s)\right)\right]
$$

$$
Y=\left(\overline{\boldsymbol{R}}^{n-2} \backslash h^{-1} \gamma\left[\bar{B}^{n-2} \times 0\right]\right) \cup h^{-1} \gamma_{1}\left[\bar{B}^{n-2} \times 0\right] .
$$

It is claimed that $Y$ is a locally $C$-flat TOP $(n-2)$-sphere in $\overline{\boldsymbol{R}}^{n}$ with $\overline{\boldsymbol{R}}^{n} \backslash Y$ homotopy equivalent to $S^{1}$.

(i) $Y$ is a TOP $(n-2)$-sphere: this follows from the fact that $\gamma\left[\bar{B}^{n-2} \times 0\right]$ is homeomorphic to $\gamma_{1}\left[\bar{B}^{n-2} \times 0\right]$ by a homeomorphism which is the identity on the boundary. Note that $\gamma_{1}\left[B^{n-2} \times 0\right] \cap X \backslash B^{n}=\emptyset$, since $\gamma_{1}\left[B^{n-2} \times 0\right] \subset \gamma_{1}\left[B^{n-2} \times B^{2}(r)\right]=$ $\gamma\left[B^{n-2} \times B^{2}(r)\right] \subset B^{n}$, so that $h^{-1} \gamma_{1}\left[B^{n-2} \times 0\right] \cap\left(\overline{\boldsymbol{R}}^{n-2} \backslash h^{-1} \gamma\left[B^{n-2} \times 0\right]\right)=\emptyset$.

(ii) $Y$ is locally $C$-flat: at points of $\overline{\boldsymbol{R}}^{n-2} \backslash h^{-1} \gamma\left[\bar{B}^{n-2} \times 0\right]$ this is immediate; at points of $h^{-1} \gamma_{1}\left[B^{n-2} \times 0\right]$ this follows from the fact that $h^{-1} \gamma_{1} \mid B^{n-2} \times B^{2}(r / 2)$ is a $C$-embedding; at the remaining points of $Y$, viz $h^{-1}\left[S^{n-3} \times 0\right]$, it follows from 
the fact that $\gamma$ and $\gamma_{t}$ are the inclusion on a neighbourhood of $S^{n-3} \times B^{2}(r)$, so that in a neighbourhood of $h^{-1}\left[S^{n-3} \times 0\right], Y$ is still $\overline{\boldsymbol{R}}^{n-2}$.

(iii) $\overline{\boldsymbol{R}}^{n} \backslash Y$ is homotopy equivalent to $S^{1}$ : in fact $\gamma_{t}$ provides an isotopy of $\overline{\boldsymbol{R}}^{n}$ throwing $\overline{\boldsymbol{R}}^{n-2}$ onto $Y$, so $Y$ is even topologically unknotted.

Now apply Theorem 3.3 of [GV] to $\left(\overline{\boldsymbol{R}}^{n}, Y\right)$. Since $n \neq 4$ or 6 , there is a $C$-homeomorphism $f:\left(\overline{\boldsymbol{R}}^{n}, \overline{\boldsymbol{R}}^{n-2}\right) \rightarrow\left(\overline{\boldsymbol{R}}^{n}, Y\right)$. Moreover, because of the way the $C$-homeomorphism was constructed in [GV], we may assume that $f$ is the identity on a neighbourhood of $\overline{\boldsymbol{R}}^{n-2} \backslash h^{-1} \gamma\left[B^{n-2} \times 0\right]$. Let $\delta=h f^{-1} h^{-1} \gamma_{1} \mid \bar{B}^{n-2} \times \bar{B}^{2}(r / 2)$. We check the required properties of $\delta$.

(a) $\delta$ is a $C$-embedding:

$$
\gamma_{1}\left[\bar{B}^{n-2} \times B^{2}(r / 2)\right] \subset \gamma_{1}\left[\bar{B}^{n-2} \times B^{2}(r)\right]=\gamma\left[\bar{B}^{n-2} \times B^{2}(r)\right] \subset h B^{n},
$$

so $h^{-1} \gamma_{1} \mid \bar{B}^{n-2} \times B^{2}(r / 2)$ is a $C$-embedding as, therefore, is $f^{-1} h^{-1} \gamma_{1} \mid \bar{B}^{n-2} \times B^{2}(r / 2)$ Making $r$ smaller if necessary, we can be sure that

$$
f^{-1} h^{-1} \gamma_{1}\left[\bar{B}^{n-2} \times B^{2}(r / 2)\right] \subset B^{n},
$$

so that $\delta$ is a well-defined $C$-embedding.

(b) $\delta$ is the identity on a neighbourhood of $S^{n-3} \times \bar{B}^{2}(r / 2)$ : this follows from the facts that $\gamma_{1}$ is the inclusion on such a set and $f$ is the identity on a neighbourhood of $\overline{\boldsymbol{R}}^{n-2} \backslash h^{-1} \gamma\left[B^{n-2} \times 0\right]$ hence on a neighbourhood of

$$
h^{-1} \gamma\left[S^{n-3} \times B^{2}(r / 2)\right]=h^{-1} \gamma_{1}\left[S^{n-3} \times B^{2}(r / 2)\right]
$$

provided $r$ is small enough.

(c) $X \cap \bar{B}^{n} \subset \delta\left[\bar{B}^{n-2} \times 0\right]$ : in fact,

$$
\begin{gathered}
f^{-1} h^{-1} \gamma_{1}\left[\bar{B}^{n-2} \times 0\right]=h^{-1} \gamma\left[\bar{B}^{n-2} \times 0\right], \\
\text { so } \delta\left[\bar{B}^{n-2} \times 0\right]=\gamma\left[\bar{B}^{n-2} \times 0\right]=X \cap \bar{B}^{n} .
\end{gathered}
$$

This completes the construction of $\delta$ and hence completes the proof of Theorem 1.

Proof of Theorem 2. The proof of Theorem 2 is much the same as that of Theorem 1 but one uses instead $(C, g)$-encasing and [GV, Theorem 3.4] neither of which requires the restriction $n \neq 6$.

\section{References}

[B] Brakes, W. R.: Quickly unknotting topological spheres. - Proc. Amer. Math. Soc. 72, 1978, 413-416.

[GV] GAULD, D. B., and J. VÄISÄL $\ddot{A}$ : Lipschitz and quasiconformal flattening of spheres and cells. - Ann. Acad. Sci. Fenn. Ser. A I 4, 1979, 371-382. 
[K] KIRBY, R. C.: Codimension two locally flat imbeddings have normal bundles. - Topology of manifolds, Markham, Chicago, 1970, 416-423.

[KS $\left.{ }_{1}\right]$ Kirby, R. C., and L. C. SiebenmanN: On the triangulation of manifolds and the Hauptvermutung. - Bull. Amer. Math. Soc. 75, 1969, 742-749.

$\left[\mathrm{KS}_{2}\right]$ KiRBy, R. C., and L. C. Siebenmann: Normal bundles for codimension 2 locally flat imbeddings. - Geometric topology, Lecture Notes in Mathematics 438, Springer-Verlag, Berlin-Heidelberg-New York, 1975, 310-324.

[M] Morse, E. E.: Affine structures in 3-manifolds. V. The triangulation theorem and Hauptvermutung. - Ann. of Math. 56, 1952, 96-114.

University of Auckland

Department of Mathematics

Auckland

New Zealand

Received 17 September 1979

Revision received 6 November 1979 